\title{
Tsallis, Rényi and Sharma-Mittal Holographic Dark Energy Models in Loop Quantum Cosmology
}

\author{
Abdul Jawad ${ }^{1}{ }^{1}$, Kazuharu Bamba ${ }^{2, *}$, Muhammad Younas ${ }^{1}$, Saba Qummer ${ }^{1}$ and Shamaila Rani ${ }^{1}$ \\ 1 Department of Mathematics, COMSATS University Islamabad, Lahore Campus, Lahore-54000, Pakistan; \\ jawadab181@yahoo.com or abduljawad@cuilahore.edu.pk (A.J.); \\ muhammadyounas@cuilahore.edu.pk (M.Y.); sabaqummer143@gmail.com (S.Q.); \\ drshamailarani@cuilahore.edu.pk (S.R.) \\ 2 Division of Human Support System, Faculty of Symbiotic Systems Science, Fukushima University, \\ Fukushima 960-1296, Japan \\ * Correspondence: bamba@sss.fukushima-u.ac.jp
}

Received: 7 October 2018; Accepted: 31 October 2018; Published: 13 Novemebr 2018

\begin{abstract}
The cosmic expansion phenomenon is being studied through the interaction of newly proposed dark energy models (Tsallis, Rényi and Sharma-Mittal holographic dark energy (HDE) models) with cold dark matter in the framework of loop quantum cosmology. We investigate different cosmic implications such as equation of state parameter, squared sound speed and cosmological plane $\left(\omega_{d}-\omega_{d}^{\prime}, \omega_{d}\right.$ and $\omega_{d}^{\prime}$ represent the equation of state (EoS) parameter and its evolution, respectively). It is found that EoS parameter exhibits quintom like behavior of the universe for all three models of HDE. The squared speed of sound represents the stable behavior of Rényi HDE and Sharma-Mittal HDE at the latter epoch while unstable behavior for Tsallis HDE. Moreover, $\omega_{d}-\omega_{d}^{\prime}$ plane lies in the thawing region for all three HDE models.
\end{abstract}

Keywords: cosmoligical parameters; dark energy models; loop quantum cosmology

\section{Introduction}

Observational data from type Ia supernovae (SNIa) [1-4], the large scale structure (LSS) [5-8] and the cosmic microwave background ( $\mathrm{CMB}$ ), anisotropies [9-11], tell us that the universe undergoes an accelerated expansion at the present time. This expanding phase of the universe is supported by an unknown component called dark energy (DE) [12-14]. The simplest candidate for DE is the cosmological constant. This model consists of a fluid with negative pressure and positive energy density. The cosmological constant suffers from some problems such as the fine-tuning problem and the coincidence problem [12]. A feasible way to relieve the cosmic coincidence problem is to suppose an interaction between dark matter and DE. The cosmic coincidence problem can also be reduced by the appropriate choice of interaction between dark matter and DE [15-17]. The nature of $\mathrm{DE}$ is mysterious and unknown. Therefore, people have suggested various models for DE such as quintessence, tachyon [18], ghost [19], K-essence [20], phantom [21], Chaplygin gas [22], polytropic gas [23,24] and holographic dark energy (HDE) [25-27].

A second approach for understanding this strange component of the universe is gravitational modification in standard theories of gravity which results in modified theories of gravity that involve some invariants depending upon specific features such as torsion, scalars, curvature etc. The several modified theories are $f(R)$ theory [28-30], where $f$ is a general differentiable function of the curvature scalar $R$, generalized teleparallel gravity, $f(T)$ [31-33] theory, contributing in the gravitational interaction through the torsion scalar T, Brans-Dicke theory, using a scalar field [34], Gauss-Bonnet theory and its modified version involving the Gauss-Bonnet invariant $G[35,36], f(R, T)$ 
theory where $T$ is the trace of the energy-momentum tensor [37], etc. For recent reviews on modified gravity theories and dark energy problem, see, for instance [14,30,38-41].

The HDE is a promising candidate of $\mathrm{DE}$, which has been studied extensively in the literature. It is based upon the holographic principle $[29,42,43]$ that states the number of degrees of freedom of a system scales with its area instead of its volume. Cohen et al. studied that the DE should obey the holographic principle and constrained by the infrared (IR) cut-off [44]. Li has examined three choices for the IR cutoff as the Hubble horizon, the future event horizon and the particle horizon and also shown that only the future event horizon is able to provide the sufficient acceleration for the universe [45]. Sheykhi [46], developed the HDE model with Hubble horizon and argued that this model possesses the ability to explain the present state of the universe with the help of interaction of DE and cold dark matter (CDM).

Hu and Ling [47] studied the relationship between interacting, HDE and cosmological parameters through observational constraints. They investigated that HDE model is justified with the present observations in the low redshift region. They also tried to reduce the cosmic coincidence problem by taking different possibilities of time rate of change of the ratio of dark matter to HDE densities for a particular choice of interacting term. Ma et al. [48] explored observational signatures of interacting and non-interacting HDE with dark matter. In these models they also observed the big rip singularity in for different parameters by using a lot of recent observational schemes. They also found that the HDE models are slightly compatible with the observations as compared to the $\Lambda$ CDM model.

In the context of thermodynamics, horizon entropy and DE can be effected by each other. Recently, due to the long-range nature of gravity, the mysterious nature of spacetime and pushed by the fact that the Bekenstein entropy is a non-extensive entropy measure. The generalized entropies, i.e., Tsallis and Rényi entropies have been assigned to the horizons to study the cosmological and gravitational phenomenon.

To study the cosmological and and gravitational phenomena many generalized entropy formalism has been applied but Tsallis and Rényi entropies generates the suitable model of universe. SharmaMittal HDE is compatible with universe expansion and whenever it is dominant in cosmos it is stable. Tsallis and Rényi entropies are attributed to the horizon to study the cosmic implications. Bekenstein entropy is also can be obtained by applying Tsallis statistics to the system. However, Tsallis and Rényi entropies can be recovered from Sharma-Mitall entropy by applying appropriate limits [49-51]. Recently, the HDE models such as Tsallis HDE [52] and Rényi HDE [53] and Sharma-Mittal HDE [54], have been studied extensively.

In classical cosmology, an important role is played by inflationary paradigm in understanding the problems of the big-bang model, by considering that the universe undergoes an expansion. However, classical general relativity (GR) fails when spacetime curvature approaches the Planck scale, due to the singularities where all physical quantities become infinite. So, the quantum gravity is considered to be necessary To interpret the circumstances in which classical (GR) breaks down [55]. In the last few decades, loop quantum gravity (LQG) has been widely applied to understand singularities in different black holes and spacetimes. LQG is not a complete theory, nor has its full stability with GR been established yet.

The loop quantum cosmology (LQC) is the application of LQG to the homogenous systems which removes the singularities. It holds the properties of a non-perturbative and background independent quantization of gravity [56]. The theory has numerous physical applications such as black hole physics and others.Recently many DE models have been studied in the context of LQC.

Here, we discuss the cosmological implications of Tsallis HDE, Rényi HDE and Sharma-Mittal HDE in the frame work of loop quantum cosmology (LQC) in the presence of the non-linear interaction between DE and dark matter [57]. This paper is organized as follows. In Section 2, we provides basics of LQC and DE models. Section 3 is devoted to cosmological parameters such as EoS parameter, cosmological plane and squared sound speed for Tsallis HDE, Rényi HDE and Sharma-Mittal HDEmodels. In the last section, we conclude the results. 


\section{Basic Equations}

In these days, DE phenomenon has been discussed in the framework of LQC to describe the quantum effects on the universe. The LQC is the effective and modern application of quantization techniques from loop quantum gravity. In the context of LQC, many DE models have been studied in last few years. In modern cosmology, the cosmic coincidence problem by taking Chaplygin gas into account with dark matter was studied by Jamil et al. [58]. Chakraborty et al. [59], explored the modified Chaplygin gas in LQC. It is also found that with the help of Loop quantum effects one can avoid the future singularities appearing in the standard cosmology. The Friedmann equation in case of LQC [60-63] is given as

$$
H^{2}=\frac{\rho_{e f f}}{3}\left(1-\frac{\rho_{e f f}}{\rho_{c}}\right)
$$

where, $H=\frac{\dot{a}}{a}$ is the Hubble parameter and dot represents the derivative of $a$, with respect to $t$ and $\rho_{e f f}=\rho_{m}+\rho_{d}, \rho_{m}$ is matter density and $\rho_{d}$ is DE density. Also, $\rho_{c}=\frac{\sqrt{3}}{16 \pi^{2} \beta^{3} G^{2} h}$ where, $\beta$ represents the dimensionless Barbero-Immirzi parameter and $\rho_{c}$ stands for critical loop quantum density [60]. The different future singularities such as big bang and big rip can be avoided in LQC. It is observed that phantom DE with the negative pressure can push the universe towards the big rip singularity where all the physical objects loose the gravitational bounds and finally get dispersed.

We consider the interacting scenario between DE and cold dark matter (CDM) and thus the energy conservation equation turns to the following equations (we refer to the reader to $[64,65]$ )

$$
\begin{aligned}
\dot{\rho}_{m}+3 H \rho_{m} & =-Q \\
\dot{\rho}_{d}+3 H\left(\rho_{d}+p_{d}\right) & =Q .
\end{aligned}
$$

The cosmological evolution of the universe was analyzed by Arevalo and Acero [66], considering a non-linear interaction term of the general form

$$
Q=3 d H \rho_{e f f}^{a+b} \rho_{m}^{c} \rho_{d}^{-b-c} .
$$

In the above equation the powers $a, b$ and $c$ characterize the interaction and $d$ is a positive coupling constant. For $(a, b, c)=(1,-1,1)$ we can get the interaction, $Q=3 d H \rho_{m}$ and for $(a, b, c)=(1,-1,0)$ one can get, $Q=3 d H \rho_{d}$. In this present work we choose the interaction is given by

$$
Q=3 d H\left(\frac{\rho_{d}^{2}}{\rho_{m}+\rho_{d}}\right)
$$

this equation correspond to the choice $(a, b, c)=(1,-2,0)$ where, $d$ is the coupling constant. The coupling of the dark matter and DE is a method to describe the evolution of the universe. The coupling constant sign decides the behavior of transformation between DE and dark matter. The positive sign indicates the decomposition of DE into dark matter while the negative sign shows the decomposition into dark matter to DE. However, the choice of positive sign of coupling constant is most favorable according to observational data. The negative sign of coupling parameter should be avoided due to the violation of laws of thermodynamics.

In this present work, we consider the power-law form of scale factor $[67,68]$ as, $a(t)=a_{0} t^{m}$ and $H(t)=\frac{m}{t}$ where, $m>0$. At different values of $m$, we have different phases of the universe

- $0<m<1$ shows the decelerated phase of the universe.

- $m=\frac{2}{3}$, corresponds to the dust dominated era.

- $m=\frac{1}{2}$, leads to the radiation dominated era.

- $m>1$, shows the accelerated phase of the universe. 
Next, we discuss the motivation and derivation of DE models.

\subsection{Tsallis Holographic Dark Energy (HDE) Model}

Li [45], has suggested the mathematical form of HDE as following constraint on its energy density $L^{3} \rho_{d} \leq L m_{p}^{2}$. This inequality can be written as

$$
\rho_{d}=\frac{3 C^{2} m_{p}^{2}}{L^{2}}
$$

where, $m_{p}{ }^{2}=(8 \pi G)^{-1}$ represents the reduced Plank mass, $C$ is a dimensionless quantity and $L$ denotes the IR cutoff. HDE density provides the relation between Ultraviolet and IR cutoff. Many IR cutoffs has been presented for explaining the accelerated expansion of the universe for example Hubble, event, particle, Granda- Oliveros, Ricci scalar etc. Tsallis and Cirto [69], studied that the horizon entropy of the black hole can be modified as $S_{\delta}=\gamma A_{\delta}$, where $\delta$ the is non-additivity parameter, $\gamma$ is an unknown constant and $A=4 \pi L^{2}$, represents the area of the horizon. Cohen et al. [44], proposed the mutual relationship between IR (L) cutoff, system entropy (S) and UV $(\Lambda)$ cut off as

$$
L^{3} \Lambda^{3} \leq(S)^{\frac{3}{4}}
$$

which leads to

$$
\Lambda^{4} \leq \gamma(4 \pi)^{\delta} L^{2 \delta-4}
$$

where, $\Lambda^{4}$ is vacuum energy density and $\rho_{d} \sim \Lambda^{4}$. So, the Tsallis HDE density [52], is given as

$$
\rho_{d}=B L^{2 \delta-4} \text {. }
$$

Here, $B$ is an unknown parameter and IR cutoff is Hubble radius which is $L=\frac{1}{H}$. The density of Tsallis HDE model using the scale factor is given as

$$
\rho_{d}=B \frac{t^{2 \delta-4}}{m^{2 \delta-4}}
$$

Inserting the value of $\rho_{d}$ along with its derivative in Equation (3) it yields expression for pressure

$$
p_{d}=\frac{1}{3} B \frac{t^{2 \delta-8}}{m^{4 \delta-3}}\left(\frac{-3 t^{4}}{m^{-2 \delta-1}}-\frac{6 B m^{5} d t^{2 \delta}}{\rho_{c}+\sqrt{\rho_{c}\left(\rho_{c}+\frac{12 m^{2}}{t^{2}}\right)}}-2 m^{2 \delta} t^{4}(\delta-2)\right) .
$$

\subsection{Rényi HDE Model}

We consider a system with $n$, states with probability distribution $P_{i}$ and satisfies the condition $\sum_{i=1}^{n} P_{i}=1$, Rényi and Tsallis entropies are well known parameters of generalized entropy is defined as

$$
S=\frac{1}{\delta} \ln \sum_{i=1}^{n} P_{i}^{1-\delta}, \quad S_{T}=\frac{1}{\delta} \sum_{i=1}^{n}\left(P_{i}^{1-\delta}-P_{i}\right),
$$

$\delta \equiv 1-U$, where, $U$ is a real parameter. Now combining above set of equations we find their mutual relation given as

$$
S=\frac{1}{\delta} \ln \left(1+\delta S_{T}\right)
$$

In Equation (13), $S$ belongs to the class of most general entropy functions of homogenous system. Recently, it is observed that Bekenstine entropy $S=\frac{A}{4}$, is in fact Tsallis entropy which gives the expression, $S=\frac{1}{\delta} \ln \left(1+\delta \frac{A}{4}\right)$, which is the Rényi entropy of the system. 
With the help of following assumption $\rho_{d} d v \propto T d s$ we can get the Rényi HDE density as

$$
\rho_{d}=\frac{3 C^{2} H^{2}}{8 \pi\left(1+\frac{\delta \pi}{H^{2}}\right)} .
$$

In our case, we suppose $8 \pi=1$ and consider the power-law scale factor we have the following expression for density

$$
\rho_{d}=\frac{3 C^{2} m^{4}}{t^{2}\left(m^{2}+\delta \pi t^{2}\right)} .
$$

The pressure for this case is also obtained from Equation (3) with the help of Equation (15)

$$
\begin{aligned}
p_{d} & =\frac{C^{2} m^{3}}{t^{4}\left(m^{2}+\pi t^{2} \delta\right)^{2}}\left(-18 C^{2} m^{5} d-\left(\rho_{c}+\sqrt{\rho_{c}\left(\rho_{c}+\frac{12 m^{2}}{t^{2}}\right)}\right)\right. \\
& \left.\times t^{2}\left(m^{2}(3 m-2)+(3 m-4) \pi t^{2} \delta\right)\right)\left(\rho_{c}+\sqrt{\rho_{c}\left(\rho_{c}+\frac{12 m^{2}}{t^{2}}\right)}\right)^{-1} .
\end{aligned}
$$

\subsection{Sharma-Mittal HDE Model}

From the Rényi entropy, we have the generalized entropy content of the system. Using Equation (12) Sharma-Mittal introduced a two parametric entropy and is defined as

$$
S_{S M}=\frac{1}{1-r}\left(\left(\sum_{i=1}^{n} P_{i}^{1-\delta}\right)^{1-r / \delta}-1\right)
$$

where $r$ is a new free parameter. We can observe that Rényi and Tsallis entropies can be recovered at the proper limits. In the limit $r \rightarrow 1$, Sharma-Mittal entropy becomes Rényi entropy while for $r \rightarrow \delta$, it is Tsallis entropy. Using Equation (12), in Equation (17) we have

$$
S_{S M}=\frac{1}{R}\left(\left(1+\delta S_{T}\right)^{R / \delta}-1\right),
$$

here, $R \equiv 1-r$. It has been recently argued that Bekenstine entropy is the proper candidate for Tsallis entropy. It allow us to replace $S_{T}$ with $S_{B}$ in above equation we have

$$
S_{S M}=\frac{1}{R}\left(\left(1+\delta \frac{A}{4}\right)^{R / \delta}-1\right) .
$$

The relation between UV $(\Lambda)$ cutoff, IR (L) cut off and and system horizon (S) is given as $\Lambda^{4} \propto \frac{S}{L^{4}}$ Now, taking $L \equiv \frac{1}{H}=\sqrt{A / 4 \pi}$, then the the energy density of DE given by

$$
\rho_{d}=\frac{3 C^{2} H^{4}}{8 \pi R}\left[\left(1+\frac{\delta \pi}{H^{2}}\right)^{R / \delta}-1\right]
$$

here, $C^{2}$ is an unknown free parameter. According to our assumptions we get the following expression for energy density

$$
\rho_{d}=\frac{3 C^{2} m^{4}}{R t^{4}}\left[\left(1+\frac{t^{2} \delta \pi}{m^{2}}\right)^{R / \delta}-1\right]
$$


The expression for pressure is obtained as

$$
\begin{aligned}
p_{d} & =\frac{C^{2} m}{t^{8}}\left(-2 \pi t^{6}\left(1+\frac{\pi t^{2} \delta}{m^{2}}\right)^{-1+R / \delta}+\left(\frac{\left(-1+\left(1+\frac{\pi t^{2} \delta}{m^{2}}\right)^{R / \delta}\right)}{R}\right)\right. \\
& \times m^{2} t^{4}(-3 m+4)-\frac{18 C^{2} m^{7} d\left(-1+\left(1+\frac{\pi t^{2} \delta}{m^{2}}\right)^{R / \delta}\right)^{2}}{R^{2}\left(\rho_{c}+\sqrt{\rho_{c}\left(\rho_{c}+\frac{12 m^{2}}{t^{2}}\right)}\right)} .
\end{aligned}
$$

\section{Cosmological Parameters}

In this section, we will discuss the physical significance of cosmological parameters such as EoS parameter, squared sound speed $v_{s}^{2}$ and $\omega_{d}-\omega_{d}^{\prime}$ plane.

\subsection{EoS Parameter}

To obtain EoS parameter we will use the following equation

$$
\omega_{d}=\frac{p_{d}}{\rho_{d}}
$$

Here, $\rho_{d}$ and $p_{d}$ represents DE density and pressure of HDE model respectively. EoS parameter is used to categorized decelerated and accelerated phases of the universe. The DE dominated phase has following eras:

- $\omega_{d}=0$ corresponds to non-relativistic matter.

- $\quad-1<\omega_{d}<-\frac{1}{3} \Rightarrow$ quintessence.

- $\omega_{d}=-1 \Rightarrow$ cosmological constant.

- $\omega_{d}<-1 \Rightarrow$ phantom.

- In this case $\omega_{d}>-1$, evolve across the boundary of cosmological constant shows the quintom behavior.

\subsubsection{For Tsallis HDE}

The EoS parameter for this model is evaluated by using Equations (10) and (11) in Equation (23)

$$
\omega_{d}=\frac{p_{d}}{\rho_{d}}=-\frac{2 B m^{-2 \delta+4} d t^{2 \delta-4}}{\rho_{c}+\sqrt{\rho_{c}\left(\rho_{c}+\frac{12 m^{2}}{t^{2}}\right)}}-\frac{2(\delta-2)}{3 m}-1 .
$$

To check the region of the universe, we plot $\omega_{d}$ versus $z$ in Figure 1. The EoS parameter exhibits the quintom-like behavior of the universe as it crosses the phantom barrier for $\delta=1.3$. However, for other values of $\delta$, it remains in the quintessence region of the universe.

\subsubsection{For Rényi HDE}

The EoS parameter for Rényi HDE is evaluated by using Equations (15) and (16) in (23) we get the following expression

$$
\begin{aligned}
\omega_{d} & =-\left(18 C^{2} m^{5} d+\left(\rho_{c}+\sqrt{\rho_{c}\left(\rho_{c}+\frac{12 m^{2}}{t^{2}}\right)}\right) t^{2}\left(m^{2}(3 m-2)\right.\right. \\
& \left.\left.+(3 m-4) \pi t^{2} \delta\right)\right) \times\left(3 m\left(\rho_{c}+\sqrt{\rho_{c}\left(\rho_{c}+\frac{12 m^{2}}{t^{2}}\right)}\right) t^{4} \delta\left(m^{2}+\pi\right)\right)^{-1} .
\end{aligned}
$$




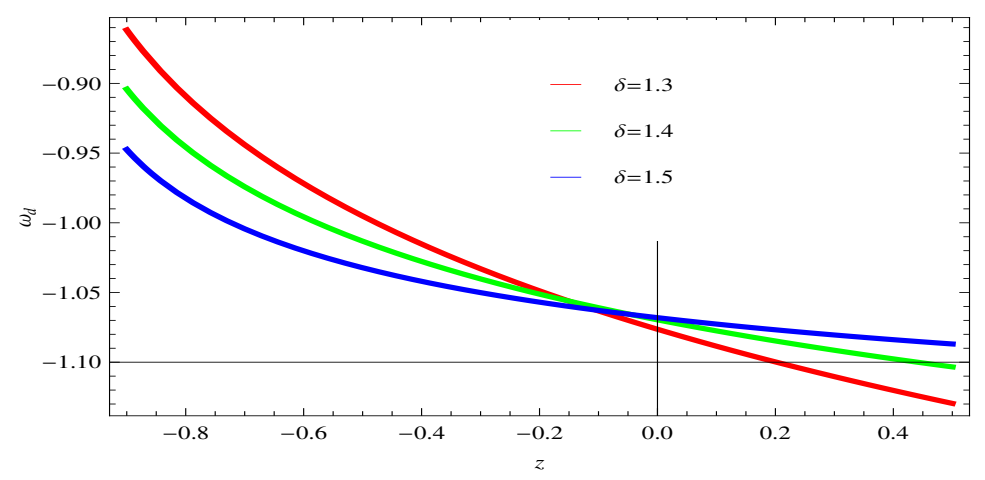

Figure 1. Plot of $\omega_{d}$ versus $z$ for Tsallis HDE at different values of $\delta$. Here $m=2, \rho_{c}=10, B=2$, $a_{0}=1, C=1, d=1$.

The plot of above parameter versus $z$ is shown in Figure 2. The trajectories of EoS parameter show the transition from phantom region to quintessence region by evolving the vacuum era of the universe. This is called quintom-like nature of the universe.

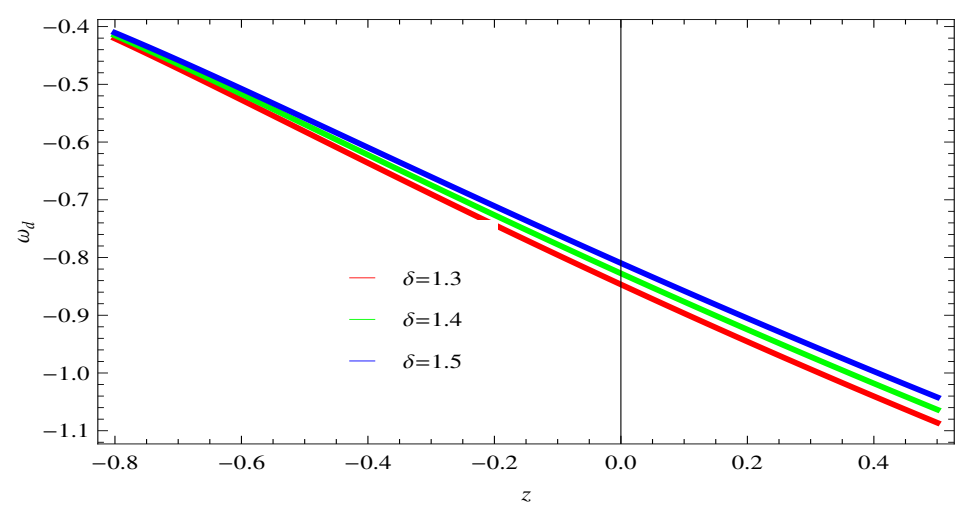

Figure 2. Plot of $\omega_{d}$ versus $z$ for Rényi HDE at different values of $\delta$. Here $m=2, \rho_{c}=10, C=1$, $a_{0}=1, d=1$.

\subsubsection{For Sharma-Mittal HDE}

The EoS for Sharma-Mittal HDE is obtained by substituting Equations (21) and (22) in Equation (23)

$$
\begin{aligned}
\omega_{d} & =R\left(-2 \pi t^{6} R\left(1+\frac{\pi t^{2} \delta}{m^{2}}\right)^{-1+R / \delta}+4 m^{2} t^{4}\left(-1+\left(1+\frac{\pi t^{2} \delta}{m^{2}}\right)^{R / \delta}\right)\right. \\
& \left.-3 m^{3} t^{4}\left(-1+\left(1+\frac{\pi t^{2} \delta}{m^{2}}\right)^{R / \delta}\right)-\frac{18 C^{2} m^{7} d\left(-1+\left(1+\frac{\pi t^{2} \delta}{m^{2}}\right)^{R / \delta}\right)^{2}}{R\left(\rho_{c}+\sqrt{\rho_{c}\left(\rho_{c}+\frac{12 m^{2}}{t^{2}}\right)}\right)}\right) \\
& \times\left(3 m^{3} t^{4}\left(-1+\left(1+\frac{\pi t^{2} \delta}{m^{2}}\right)^{R / \delta}\right)\right)^{-1} .
\end{aligned}
$$

In Figure 3, the curves of EoS parameter shows quintom-like behavior of the universe. 


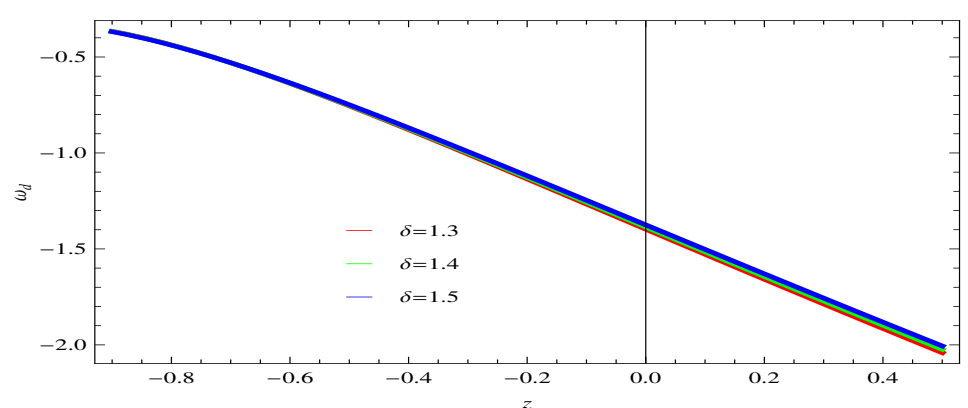

Figure 3. Plot of $\omega_{d}$ versus $z$ for Sharma-Mittal HDE at different values of $\delta$. Here $m=2, \rho_{c}=10$, $C=1, a_{0}=1, d=1, R=-2$.

\subsection{Stability Analysis}

To analyze the stability of the HDE models in LQC scenario we evaluate the squared sound speed which is given by

$$
v_{s}^{2}=\frac{d p_{d}}{d \rho_{d}}=\frac{d p_{d} / d t}{d \rho_{d} / d t}
$$

The sign of $v_{s}^{2}$ determines the stability of HDE model. For $v_{s}^{2}>0$, the model is stable otherwise it is unstable.

\subsubsection{For Tsallis HDE}

The expression for squared sound speed can be obtained by taking the derivative of Equations (10) and (11) with respect to $t$, and then substitute in Equation (27) we have

$$
\begin{aligned}
v_{s}^{2} & =\frac{m^{-2 \delta-1}}{6 t^{4}(\delta-2)}\left(-\frac{\left.72 B m^{7} d \sqrt{\rho_{c}\left(\rho_{c}+\frac{12 m^{2}}{t^{2}}\right.}\right) t^{2 \delta}}{\left(\rho_{c}+\sqrt{\rho_{c}\left(\rho_{c}+\frac{12 m^{2}}{t^{2}}\right)}\right)^{2}\left(12 m^{2}+\rho_{c} t^{2}\right)}\right. \\
& \left.-6 m^{2 \delta+1} t^{4}(\delta-2)-\frac{24 B m^{5} d t^{2 \delta}(\delta-2)}{\rho_{c}+\sqrt{\rho_{c}\left(\rho_{c}+\frac{12 m^{2}}{t^{2}}\right)}}-4 m^{2 \delta} t^{4}(\delta-2)^{2}\right) .
\end{aligned}
$$

Figure 4 shows the graph between $v_{s}^{2}$ and $z$. This graph is used to analyze the stability of the Tsallis HDE model under different parametric values. From the figure one can see that $v_{s}{ }^{2}<0$ at the early, present and latter epoch. Hence this model shows unstable behavior at the present, early and latter epoch.

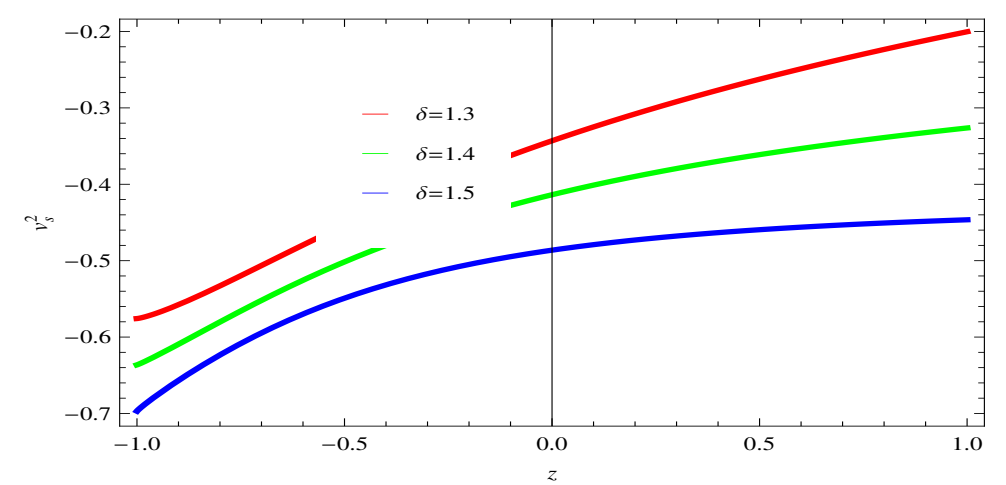

Figure 4. Plot of $v_{s}^{2}$ versus $z$ for Tsallis HDE at different values of $\delta$. Here $m=1.1, \rho_{c}=10, C=1$, $B=-1.5, a_{0}=1, d=1$. 


\subsubsection{For Rényi HDE}

The expression of squared sound speed for Rényi HDE model can be obtained by using Equation (27) is given by

$$
\begin{aligned}
v_{s}^{2} & =-\left(2 \rho _ { c } \left(t ^ { 2 } \left(12 \rho_{c} m^{2}+6 m^{2} \sqrt{\rho_{c}\left(\rho_{c}+\frac{12 m^{2}}{t^{2}}\right)}+\rho_{c}^{2} t^{2}+\rho_{c} t^{2}\right.\right.\right. \\
& \left.\times \sqrt{\rho_{c}\left(\rho_{c}+\frac{12 m^{2}}{t^{2}}\right)}\right)\left(m^{4}(-2+3 m)+3 m^{2}(-2+3 m) \pi t^{2} \delta\right. \\
& \left.+2(3 m-4) \pi^{2} t^{4} \delta^{2}\right)+18 c^{2} m^{5} d\left(2 \pi t^{4} \delta\left(\rho_{c}+\sqrt{\rho_{c}\left(\rho_{c}+\frac{12 m^{2}}{t^{2}}\right)}\right)\right. \\
& \left.\left.+9 m^{4}+m^{2} t^{2}\left(\rho_{c}+\sqrt{\rho_{c}\left(\rho_{c}+\frac{12 m^{2}}{t^{2}}\right)}+21 \pi \delta\right)\right)\right)\left(3 m t ^ { 4 } \left(2 \pi t^{2} \delta\right.\right. \\
& \left.\left.+m^{2}\right)\left(\rho_{c}+\sqrt{\rho_{c}\left(\rho_{c}+\frac{12 m^{2}}{t^{2}}\right)}\right)^{2}\left(m^{2}+\pi t^{2} \delta\right) \sqrt{\rho_{c}\left(\rho_{c}+\frac{12 m^{2}}{t^{2}}\right)}\right)^{-1} .
\end{aligned}
$$

In the present model, we significantly investigate the stability analysis of the Rényi HDE model which depends upon the different cosmological parameters. Here we take some specific values $\rho_{c}=10, C=1, d=1$ for different values of $\delta$. In Figure 5, the curves for $v_{s}^{2}$ shows the positive behavior for different values of $\delta$ at latter epoch which shows the stability the Rényi HDE model at the latter epoch.

\subsubsection{For Sharma-Mittal HDE}

Using Equation (27) and after some calculations we obtained the expression for squared sound speed which is given by

$$
\begin{aligned}
v_{s}^{2} & =\left(2 C ^ { 2 } m \left(3 \pi t^{2}(2-m)\left(1+\frac{\pi t^{2} \delta}{m^{2}}\right)^{-1+R / \delta}+\frac{2 m^{2} \pi^{2} t^{4}(-R+\delta)}{\left(m^{2}+\pi t^{2} \delta\right)^{2}}\right.\right. \\
& \left.\times\left(1+\frac{\pi t^{2} \delta}{m^{2}}\right)^{R / \delta}\right)+\frac{2 m^{2}}{R}(3 m-4)\left(-1+\left(1+\frac{\pi t^{2} \delta}{m^{2}}\right)^{R / \delta}\right) \\
+ & \frac{72 C^{4} m^{6} d}{\left(\rho_{c} R+R \sqrt{\rho_{c}\left(\rho_{c}+\frac{12 m^{2}}{t^{2}}\right)}\right)^{2} t^{9}}\left(\rho _ { c } \left(\pi R t^{2}\left(1+\frac{\pi t^{2} \delta}{m^{2}}\right)^{-1+R / \delta}\right.\right. \\
& \left.\times\left(1-\left(1+\frac{\pi t^{2} \delta}{m^{2}}\right)^{R / \delta}\right)+2 m^{2}\left(-1+\left(1+\frac{\pi t^{2} \delta}{m^{2}}\right)^{R / \delta}\right)^{2}\right) \\
& +\sqrt{\rho_{c}\left(\rho_{c}+\frac{12 m^{2}}{t^{2}}\right)}\left(\frac{m^{2}\left(21 m^{2}+2 \rho_{c} t^{2}\right)\left(-1+\left(1+\frac{\pi t^{2} \delta}{m^{2}}\right)^{R / \delta}\right)^{2}}{12 m^{2}+\rho_{c} t^{2}}\right. \\
& \left.\left.\left.+\pi R t^{2}\left(1+\frac{\pi t^{2} \delta}{m^{2}}\right)^{-1+R / \delta}\left(1-\left(1+\frac{\pi t^{2} \delta}{m^{2}}\right)^{R / \delta}\right)\right)\right)\right)^{\left(6 C^{2} m^{2} \pi t^{2}\right.} \\
& \left.\times\left(1+\frac{\pi t^{2} \delta}{m^{2}}\right)^{-1+R / \delta}-\frac{12 C^{2} m^{4}}{R}\left(-1+\left(1+\frac{\pi t^{2} \delta}{m^{2}}\right)^{R / \delta}\right)\right)^{-1} \cdot
\end{aligned}
$$




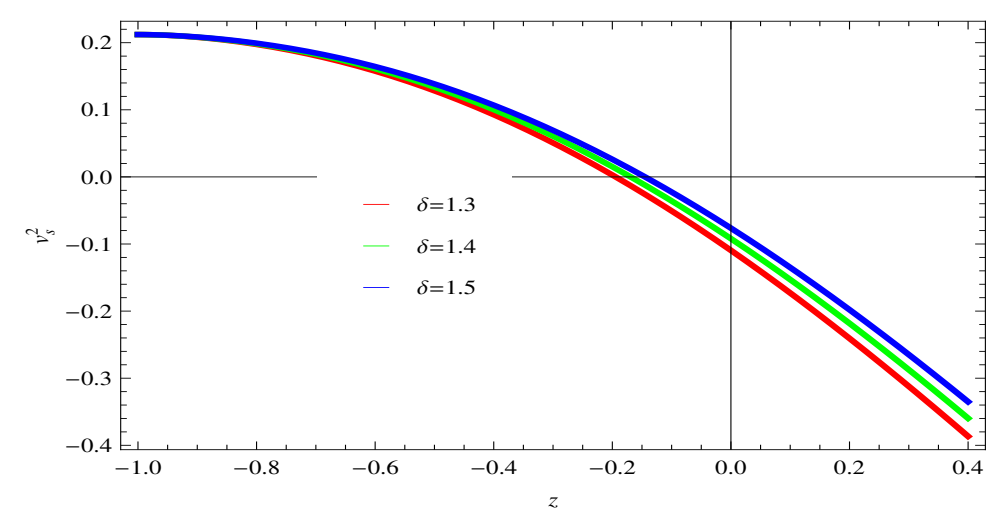

Figure 5. Plot of $v_{s}^{2}$ versus $z$ for Rényi HDE at different values of $\delta$. Here $m=1.1, \rho_{c}=10, C=1$, $a_{0}=1, d=1$.

To check the stability of the Sharma-Mittal HDE model we plot a graph of $v_{s}^{2}$ against $z$. In Figure 6, the curves for $v_{s}^{2}$ shows the positive behavior for different values of $\delta$ at latter epoch which shows the stability the Sharma-Mittal HDE model at the latter epoch.

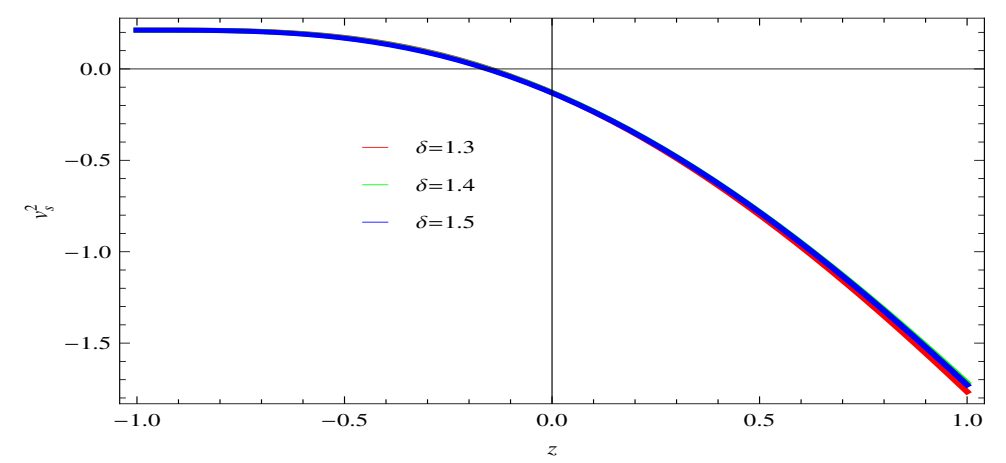

Figure 6. Plot of $v_{s}^{2}$ versus $z$ for Sharma-Mittal HDE at different values of $\delta$. Here $m=2.5, \rho_{c}=10$, $C=1, d=1, R=-2,3$ and 4 .

\section{3. $\omega_{d}-\omega_{d}^{\prime}$ Plane}

Caldwell and Linder [70], proposed the $\omega_{d}-\omega_{d}^{\prime}$ plane to explain the dynamical property of DE model in quintessence scalar field. Here, $\omega_{d}$ is EoS parameter and $\omega_{d}^{\prime}$ is its evolutionary form where prime denotes the derivative with respect to $\ln a$. They divided the $\omega_{d}-\omega_{d}^{\prime}$ plane in two parts, the thawing part $\left(\omega_{d}<0, \omega_{d}^{\prime}>0\right)$ is the region where EoS parameter nearly evolves from $\omega_{d}<-1$, increases with time while its evolution parameter expresses positive behavior, and the freezing part $\left(\omega_{d}<0, \omega_{d}^{\prime}<0\right)$ is the evolution parameter for EoS parameter remains negative.

\subsubsection{For Tsallis HDE}

The expression $\omega_{d}^{\prime}$ for THDE can be obtained by taking the derivative of Equation (24) with respect to $\ln a$ for THDE.

$$
\begin{aligned}
\omega_{d}^{\prime} & =4 \rho_{c} B m^{-2 \delta+3} d t^{2 \delta-4}\left(\left(\rho_{c}+\sqrt{\rho_{c}\left(\rho_{c}+\frac{12 m^{2}}{t^{2}}\right)}\right)(\delta-2)+6 m^{2}(2 \delta-3)\right) \\
& \times\left(\left(\rho_{c}+\sqrt{\rho_{c}\left(\rho_{c}+\frac{12 m^{2}}{t^{2}}\right)}\right)^{2} \sqrt{\rho_{c}\left(\rho_{c}+\frac{12 m^{2}}{t^{2}}\right)}\right)^{-1} .
\end{aligned}
$$


In Figure $7, \omega_{d}-\omega_{d}^{\prime}$, plane is used to check the region for this Tsallis HDE model. It can be seen that the value of $\omega_{d}^{\prime}$ decreases as we increase the value of $\omega_{d}$. We can see that $\omega_{d}<0$ and $\omega_{d}^{\prime}>0$ for all values of $\delta$, which corresponds to the thawing region of the universe.

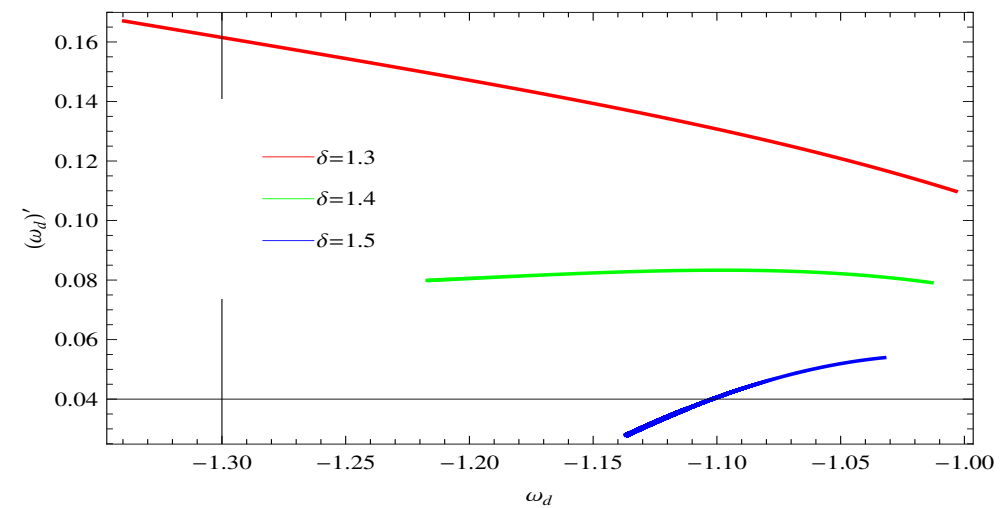

Figure 7. Plot of $\omega_{d}$ versus $\omega_{d}^{\prime}$ for Tsallis HDE at different values of $\delta$. Here $m=2, \rho_{c}=10, C=1$, $B=2, a_{0}=1, d=1$.

\subsubsection{For Rényi HDE}

The expression of $\omega_{d}^{\prime}$ for Rényi HDE can be obtained by taking the derivative of EoS parameter in Equation (25) with respect to $\ln a$ for Rényi HDE.

$$
\begin{aligned}
\omega_{d}^{\prime} & =4 \rho_{c} \pi t^{2}\left(12 m^{2}+\rho_{c} t^{2}\right) \delta+3 c^{2} m d\left(6 m^{4} \sqrt{\rho_{c}\left(\rho_{c}+\frac{12 m^{2}}{t^{2}}\right)}+\right. \\
& +6 m^{2} \pi\left(-2 \rho_{c}+3 \sqrt{\rho_{c}\left(\rho_{c}+\frac{12 m^{2}}{t^{2}}\right)}\right) t^{2} \delta+\rho_{c} \pi t^{4} \delta\left(-\rho_{c}\right. \\
& \left.+\sqrt{\left.\rho_{c}\left(\rho_{c}+\frac{12 m^{2}}{t^{2}}\right)\right)}\right) \times\left(3 \rho_{c}\left(12 m^{2}+\rho_{c} t^{2}\right)\left(m^{2}+\pi t^{2} \delta\right)^{2}\right)^{-1}
\end{aligned}
$$

In Figure 8, we find the region on the $\omega_{d}-\omega_{d}^{\prime}$, for the model under consideration. In this plane, the EoS parameter corresponds to the quintessence era, also $\omega_{d}-\omega_{d}^{\prime}$ shows that $\left(\omega_{d}<0, \omega_{d}^{\prime}>0\right.$ which leads to the thawing region.

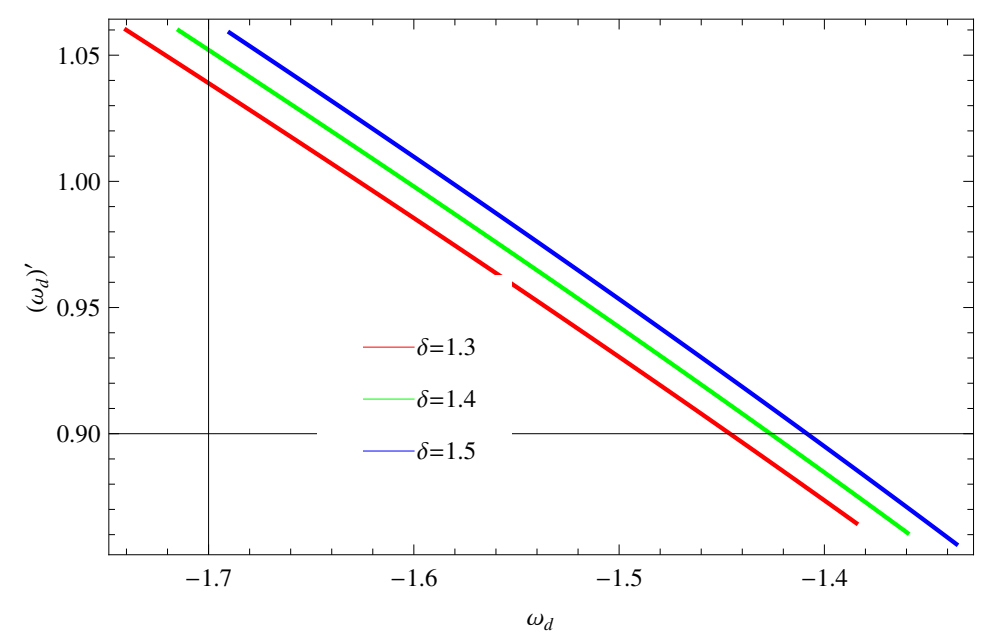

Figure 8. Plot of $\omega_{d}$ versus $\omega_{d}^{\prime}$ for Rényi HDE at different values of $\delta$. Here $m=2, \rho_{c}=10, a_{0}=1$, $C=1, d=1$. 


\subsubsection{For Sharma-Mittal HDE}

The expression of $\omega_{d}^{\prime}$ for Sharma-Mittal HDE can be obtained by taking the derivative of Equation (26) with respect to $\ln a$ for Sharma-Mittal HDE.

$$
\begin{aligned}
\omega_{d}^{\prime} & =-\frac{4 \pi R t^{2}\left(1+\frac{\pi t^{2} \delta}{m^{2}}\right)^{R / \delta}\left(-\pi R t^{2}+m^{2}\left(-1+\left(1+\frac{\pi t^{2} \delta}{m^{2}}\right)^{R / \delta}\right)\right)}{3 m^{2}\left(m^{2}+\pi t^{2} \delta\right)^{2}\left(-1+\left(1+\frac{\pi t^{2} \delta}{m^{2}}\right)^{R / \delta}\right)^{2}} \\
& +12 C^{2} m^{3} d\left(\rho_{c}\left(-2+\frac{\left(2 m^{2}-\pi t^{2}(R-2 \delta)\right)\left(1+\frac{\pi t^{2} \delta}{m^{2}}\right)^{R / \delta}}{m^{2}+\pi t^{2} \delta}\right)\right. \\
& +\frac{\sqrt{\rho_{c}\left(\rho_{c}+\frac{12 m^{2}}{t^{2}}\right)}}{\left(12 m^{2}+\rho_{c} t^{2}\right)\left(m^{2}+\pi t^{2} \delta\right)}\left(-2\left(9 m^{2}+\rho_{c} t^{2}\right)\left(m^{2}+\pi t^{2} \delta\right)\right. \\
& \left.\left.+\left(1+\frac{\pi t^{2} \delta}{m^{2}}\right)^{R / \delta}\left(18 m^{4}-\rho_{c} \pi t^{4}(R-2 \delta)+2 m^{2} t^{2}\left(\rho_{c}-6 \pi R+9 \pi \delta\right)\right)\right)\right) \\
& \times\left(R\left(\rho_{c}+\sqrt{\rho_{c}\left(\rho_{c}+\frac{12 m^{2}}{t^{2}}\right)}\right)^{2} t^{4}\right)^{-1} .
\end{aligned}
$$

To find out the region of the $\omega_{d}-\omega_{d}^{\prime}$, for the model which is under consideration we construct the $\omega_{d}-\omega_{d}^{\prime}$ plane for different parametric values. In Figure 9 we can see that $\omega_{d}-\omega_{d}^{\prime}$ shows that $\left(\omega_{d}<0, \omega_{d}^{\prime}>0\right)$ which corresponds to the thawing region.

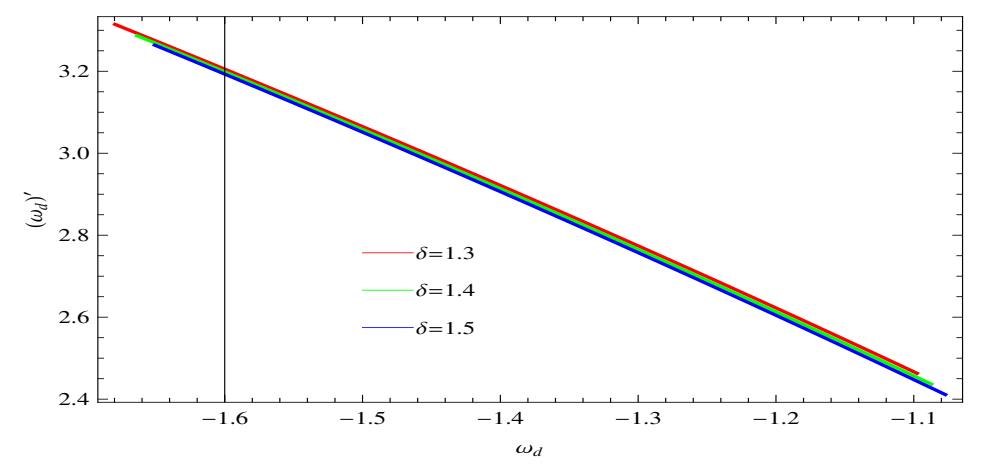

Figure 9. Plot of $\omega_{d}^{\prime}$ versus $\omega_{d}$ for Sharma-Mittal HDE at different values of $\delta$. Here $m=\frac{2}{3}, \rho_{c}=10$, $C=1, a_{0}=1, d=1, R=-2$

\section{Concluding Remarks}

In this paper, cosmological implications with the help of non-linear interaction terms between dark matter and DE models have been discussed in the framework of LQC. For this purpose, we have constructed the EoS parameter, the squared sound speed and $\omega_{d}-\omega_{d}^{\prime}$ plane and discussed their graphical behavior.

- The trajectories of EoS parameter in all three models HDE exhibit the quintom-like nature of the universe as it shows transition of the universe from phantom era (at early and present) towards quintessence era (latter epoch) by evolving phantom barrier.

- To analyze the stability of the Tsallis HDE, Rényi HDE and Sharma-Mittal HDE models we check the graphical behavior of squared sound speed. For Tsallis HDE model, it is observed that $v_{s}{ }^{2}<0$ for all values of $z$ which leads to the instability of this model. On the other hand, for Rényi HDE, the squared speed of sound shows unstable behavior at the early and present epoch while leads 
to the stability at the latter epoch. The same behavior of the squared speed of sound has been observed in case of Sharma-Mittal HDE model.

- Also, $\omega_{d}-\omega_{d}^{\prime}$ corresponds to thawing region $\left(\omega_{d}<0\right.$ and $\left.\omega_{d}^{\prime}>0\right)$ for all three models of HDE.

Author Contributions: A.J. has given the idea about this topic and drafted this article, calculations have been done by M.Y. and S.Q. Furthermore, graphs were drawn by S.R. and proofreading has been conducted by K.B.

Funding: This research received no external funding.

Conflicts of Interest: The authors declare no conflict of interest.

\section{References}

1. Riess, A.G.; Filippenko, A.V.; Challis, P.; Clocchiattia, A.; Diercks, A.; Garnavich, P.M.; Gilliland, R.L.; Hogan, C.J.; Jha, S.; Kirshner, R.P.; et al. Observational evidence from supernovae for an accelerating Universe and a cosmological constant. Astron. J. 1998, 116, 1009-1038. [CrossRef]

2. Perlmutter, S.; Aldering, G.; Goldhaber, G.; Knop, R.A.; Nugent, P.; Castro, P.G.; Deustua, S.; Fabbro, S.; Goobar, A.; Groom, D.E.; et al. Measurements of Omega and Lambda from 42 High-Redshift Supernovae. Astrophys. J. 1999, 517, 565-586. [CrossRef]

3. Bernardis, P.; Ade, P.A.R.; Bock, J.J.; Bond, J.R.; Borrill, J.; Boscaleri, A.; Coble, K.; Crill, B.P.; DeGasperis, G.; Farese, P.C.; et al. A flat Universe from high-resolution maps of the cosmic microwave background radiation. Nature 2000, 404, 955-959. [CrossRef] [PubMed]

4. Perlmutter, S.; Aldering, G.; Amanullah, R.; Astier, P.; Blanc, G.; Burns, M.S.; Conley, A.; Deustua, S.E.; Doi, M.; Ellis, R.; et al. New Constraints on and w from an Independent Set of 11 High-Redshift Supernovae Observed with the Hubble Space Telescope. Astrophys. J. 2003, 598, 102-137.

5. Colless, M.; Dalton, G.; Maddox, S.; Sutherland, W.; Norberg, P.; Cole, S.; Hawthrone, J.B.; Bridges, T.; Cannon, R.; Collins, C.; et al. The 2dF Glaxy Redshift Survey: Spectra and redshift. Mon. Not. R. Astron. Soc. 2001, 328, 1039-1063. [CrossRef]

6. Tegmark, M.; Strauss, M.A.; Blanton, M.R.; Abazajian, K.; Dodelson, S.; Sandvik, H.; Wang, X.; Weinberg, D.H.; Zehavi, I.; Bahcall, N.A.; et al. Cosmological parameters from SDSS and WMAP. Phys. Rev. D 2004, 69, 103501-103526. [CrossRef]

7. Cole, S.; Percival, W.J.; Peacock, R.A.; Norberg, P.; Baugh, C.M.; Frenk, C.S.; Baldry, I.; Bland-Hawthorn, J.; Bridges, T.; Cannon, R.; et al. The 2dF Galaxy Redshift Survey: Power-spectrum analysis of the final dataset and cosmological implications. Mon. Not. R. Astron. Soc. 2005, 362, 505-534. [CrossRef]

8. Springel, V.; Frenk, C.S.; White, S.M.D. The large-scale structure of the Universe. Nature 2006, 440, 1137-1144. [CrossRef] [PubMed]

9. Hanany, S.; Ade, P.; Balbi, A.; Bock, J.; Borrill, J.; Boscaleri, A.; Bernardis, P.; Ferreira, P.G.; Hristov, V.V.; Jaffe, A.H.; et al. MAXIMA-1: A Measurement of the Cosmic Microwave Background Anisotropy on angular scales of 10 arcminutes to 5 degrees. Astrophys. J. Lett. 2000, 545, L5-L9. [CrossRef]

10. Netterfield, C.B.; Ade, P.A.R.; Bock, J.J.; Bond, J.R.; Borrill, J.; Boscaleri, A.; Coble, K.; Contaldi, C.R.; Crill, B.P.; Bernardis, P.; et al. A measurement by BOOMERANG of multiple peaks in the angular power spectrum of the cosmic microwave background. Astrophys. J. 2002, 571, 604-614. [CrossRef]

11. Spergel, D.N.; Verde, L.; Peiris, H.V.; Komatsu, E.; Nolta, M.R.; Bennett, C.L.; Halpern, M.; Hinshaw, G.; Jarosik, N.; Kogut, A.; et al. First Year Wilkinson Microwave Anisotropy Probe (WMAP) Observations: Determination of Cosmological Parameters. Astrophys. J. Suppl. 2003, 148, 175-194. [CrossRef]

12. Roos, M. Introduction to Cosmology; John Wiley and Sons: Chichester, UK, 2003.

13. Nojiri, S.; Odintsov, S.D. The new form of the equation of state for dark energy fluid and accelerating universe. Phys. Lett. B 2006, 639, 144-150. [CrossRef]

14. Bamba, K.; Capozziello, S.; Nojiri, S.; Odintsov, S.D. Dark energy cosmology: The equivalent description via different theoretical models and cosmography tests. Astrophys. Space Sci. 2012, 342, 155-228. [CrossRef]

15. Zimdahl, W.; Pavon, D. Scaling Cosmology. Gen. Rel. Grav. 2003, 35, 413-422. [CrossRef]

16. Del Campo, S.; Herrera, R.; Olivares, G.; Pavon, D. Interacting models of soft coincidence. Phys. Rev. D 2006, 74, 023501-9. [CrossRef]

17. Sadjadi, H.M.; Alimohammadi, M. Cosmological coincidence problem in interacting dark energy models. Phys. Rev. D 2006, 74, 103007-7. [CrossRef] 
18. Setare, M.R. The holographic dark energy in non-flat Brans-Dicke cosmology. Phys. Lett. B 2007, 644, 99-103. [CrossRef]

19. Malekjani, M.; Naderi, T.; Pace, F. Effects of ghost dark energy perturbations on the evolution of spherical overdensities. MNRAS B 2015, 453, 4148-4158. [CrossRef]

20. Chiba, T.; Okabe, T.; Yamaguchi, M. Kinetically driven quintessence. Phys. Rev. D 2000, 62, 023511-023518. [CrossRef]

21. Nojiri, S.; Odintsov, S.D. Quantum de Sitter cosmology and phantom matter. Phys. Lett. B 2003, 562, 147-152. [CrossRef]

22. Pasquier, V.; Moschella, U.; Kamenshchick, A.Y.; Moschella, U.; Pasquier, V. An alternative to quintessence. Phys. Lett. B 2001, 511, 265-268.

23. Kleidis, K.; Spyrou, N.K. Polytropic dark matter flows illuminate dark energy and accelerated expansion. Astron. Astrographys. 2015, 576, A23. [CrossRef]

24. Kleidis, K.; Spyrou, N.K. Dark Energy: The Shadowy Reflection of Dark Matter. Entropy 2016, 18, 94. [CrossRef]

25. Weinberg, S. The cosmological constant problem. Rev. Mod. Phys. 1998, 61, 1-20. [CrossRef]

26. Copeland, E.J.; Sami, M.; Tsujikawa, S. Dynamics of dark energy. Int. J. Mod. Phys. D 2006, 15, $1753-1935$. [CrossRef]

27. Padmanabhan, T. Cosmological Constant-The Weight of the Vacuum. Phys. Rep. 2003, 380, $235-320$. [CrossRef]

28. Felice, A.D.; Tsujikawa, S. $f(R)$ Theories. Living Rev. Relativ. 2010, 13, 3-163. [CrossRef] [PubMed]

29. Sahni, V. The Cosmological Constant Problem and Quintessence. Class. Quant. Grav. 2002, 19, 3435-3448. [CrossRef]

30. Nojiri, S.; Odintsov, S.D. Unified cosmic history in modified gravity: From $F(R)$ theory to Lorentz non-invariant models. Phys. Rep. 2011, 505, 59-144. [CrossRef]

31. Linder, E.V. Einstein's other gravity and the acceleration of the Universe. Phys. Rev. D 2010, 81, 127301-127303. [CrossRef]

32. Ferraro, R.; Fiorini, F. Non-trivial frames for $f(T)$ theories of gravity and beyond. Phys. Lett. B 2011, 702, 75-80. [CrossRef]

33. Sharif, M.; Rani, S. Wormhole solutions in $f(T)$ gravity with noncommutative geometry. Phys. Rev. D 2013, 88, 123501-123510. [CrossRef]

34. Brans, C.H.; Dicke, R.H. Mach's Principle and a Relativistic Theory of Gravitation. Phys. Rev. D 1961, 124, 925-935. [CrossRef]

35. Nojiri, S.; Odintsov, S.D. Modified Gauss-Bonnet theory as gravitational alternative for dark energy. Phys. Lett. B 2005, 631, 1-6. [CrossRef]

36. Cognola, G.; Elizalde, E.; Nojiri, S.; Odintsov, S.D.; Zerbini, S. Dark energy in modified Gauss-Bonnet gravity: Late-time acceleration and the hierarchy problem. Phys. Rev. D 2006, 73, 084007-084022. [CrossRef]

37. Harko, T.; Francisco, S.N.; Lobo, F.S.N.; Nojiri, S.; Odintsov, S.D. $f(R, T)$ gravity. Phys. Rev. D 2011, 84 , 024020-024031. [CrossRef]

38. Capozziello, S.; De Laurentis, M. Extended Theories of Gravity. Phys. Rep. 2011, 509, 167-321. [CrossRef]

39. Capozziello, S.; Faraoni, V. Beyond Einstein Gravity: A Survey of Gravitational Theories for Cosmology and Astrophysics, 1st ed.; Springer: Dordrecht, The Netherlands, 2010; Volume 170.

40. Bamba, K.; Odintsov, S. Inflationary cosmology in modified gravity theories. Symmetry 2015, 7, $220-240$. [CrossRef]

41. Nojiri, S.; Odintsov, S.D.; Oikonomou, V.K. Modified Gravity Theories on a Nutshell: Inflation, Bounce and Late-time Evolution. Phys. Rep. 2017, 692, 1-104. [CrossRef]

42. Susskind, L. The World as a Hologram. J. Math. Phys. 1995, 36, 6377-6396. [CrossRef]

43. Nojiri, S.; Odintsov, S.D. Unifying phantom inflation with late-time acceleration: Scalar phantom nonphantom transition model and generalized holographic dark energy. Gen. Rel. Grav. 2006, 38, 1285-1304. [CrossRef]

44. Cohen, A.G.; Kaplan, D.B.; Nelson, A.E. Effective Field Theory, Black Holes, and the Cosmological Constant. Phys. Rev. Lett. 1999, 82, 4971-4974. [CrossRef]

45. Li, M. A Model of Holographic Dark Energy. Phys. Lett. B 2004, 603, 1-5. [CrossRef]

46. Sheykhi, A. Holographic scalar field models of dark energy. Phys. Rev. D 2011, 84, 107302-107306. [CrossRef] 
47. Hu, B.; Ling, Y. Interacting dark energy, holographic principle, and coincidence problem. Phys. Rev. D 2006, 73, 123510-123518. [CrossRef]

48. Ma, Y.Z.; Gong, Y.; Chen, X. Features of holographic dark energy under combined cosmological constraints. Eur. Phys. J. C 2009, 60, 303-315. [CrossRef]

49. Tsallis, C. Possible generalization of Boltzmann-Gibbs statistics. J. Stat. Phys. 1988, 52, 479-487. [CrossRef]

50. Rényi, A . On measures of entropy and Information. In Proceedings of the 4th Berkely Symposium on Mathematics, Statistics and Probability; University California Press: Berkeley, CA, USA, 1961; Volume 1, pp. 547-561.

51. Sharma, B.D.; Mittal, D.P. New nonadditive measures of entropy for discrete probability distributions. J. Math. Sci. 1975, 10, 28-40.

52. Tavayef, M.; Sheykhi, A.; Bamba, K.; Moradpour, H. Tsallis holographic dark energy. Phys. Lett. B 2018, 781, 195-200. [CrossRef]

53. Moradpour, H.; Moosavi, S.A.; Lobo, I.P.; Graca, J.P.M.; Jawad, A.; Salako, I.G. Thermodynamic approach to holographic dark energy and the Renyi entropy. Eur. Phys. J. C 2018, 829, 78-83. [CrossRef]

54. Jahromi, A.S.; Moosavi, S.A.; Moradpour, H.; Grac, J.P.M.; Lobo, I.P.; Salako, I.G.; Jawad, A. Generalized entropy formalism and a new holographic dark energy model. Phys. Lett. B 2018, 780, 21-24. [CrossRef]

55. Bojowald, M. Loop Quantum Cosmology. Living Rev. Relativ. 2008, 11, 4-134. [CrossRef] [PubMed]

56. Rovelli, C. Loop Quantum Gravity. Living Rev. Relativ. 2008, 11, 5-73. [CrossRef] [PubMed]

57. Oliveros, A. Slow-roll inflation from massive vector fields non-minimally coupled to gravity. Astrphys. Space Sci. 2017, 362, 1-6. [CrossRef]

58. Jamil, M.; Debnath, U. Interacting modified Chaplygin gas in loop quantum cosmology. Astrophys. Space Sci. 2011, 333, 3-8. [CrossRef]

59. Chakraborty, S.; Debnath, U.; Ranjit, C. Observational constants of modified Chaplygin gas in loop quantum cosmology. Eur. Phys. J. C 2012, 72, 2101-2108. [CrossRef]

60. Ashtekar, A. Albert Einstein Century International conference. AIP Conf. Proc. 2006, 861, 3-14.

61. Ashtekar, A.; Pawlowski, T.; Singh, P. Quantum Nature of the Big Bang: Improved dynamics. Phys. Rev. D 2006, 74, 084003-084042. [CrossRef]

62. Singh, P. Loop cosmological dynamics and dualities with Randall-Sundrum braneworlds. Phys. Rev. D 2006, 73, 063508-063516. [CrossRef]

63. Sami, M.; Singh, P.; Tsujikawa, S. Avoidance of future singularities in loop quantum cosmology. Phys. Rev. D 2006, 74, 043514-043519. [CrossRef]

64. Zimdahl, W.; Pavon, D.; Chimento, L.P. Interacting Quintessence. Phys. Lett. B 2001, 521, 133-138. [CrossRef]

65. Li, M.; Li, X.; Wang, S.; Wang, Y. Dark Energy. Commun. Theor. Phys. 2011, 56, 525-604. [CrossRef]

66. Arevalo, F.; Bacalhau, A.P.R.; Zimdahl, W. Cosmological dynamics with non-linear interactions. Class. Quant. Grav. 2012, 29, 235001-235023. [CrossRef]

67. Sharif, M.; Zubair, M. Energy Conditions Constraints and Stability of Power Law Solutions in $f(R, T)$ Gravity. J. Phys. Soc. Jpn. 2013, 82, 014002-014010. [CrossRef]

68. Sharif, M.; Zubair, M. Cosmological reconstruction and stability in $f(R, T)$ gravity. Gen. Relativ. Gravit. 2014, 46, 1723-1752. [CrossRef]

69. Tsallis, C.; Citro, L.J.L. Black hole thermodynamical entropy. Eur. Phys. J. C 2013, 73, 2487-2493. [CrossRef]

70. Caldwell, R.R.; Linder, E.V. Limits of Quintessence. Phys. Rev. Lett. 2005, 95, 141301-141304. [CrossRef] [PubMed]

(C) 2018 by the authors. Licensee MDPI, Basel, Switzerland. This article is an open access article distributed under the terms and conditions of the Creative Commons Attribution (CC BY) license (http:// creativecommons.org/licenses/by/4.0/). 